\title{
Determinations of phosphorus in fertilizers by spark discharge-assisted laser-induced breakdown spectroscopy
}

\author{
Alan Lima Vieira ${ }^{a}$, Tiago Varão Silva ${ }^{a}$, Felipe Schiavon Inocêncio de Sousa ${ }^{b}$, Giorgio Saverio Senesi ${ }^{c}$, \\ Dário Santos Júnior ${ }^{\mathrm{d}}$, Edilene Cristina Ferreira ${ }^{a}$, José Anchieta Gomes Neto ${ }^{\mathrm{a}, *}$ \\ a São Paulo State University - UNESP, Analytical Chemistry Department, P.O. Box 355, 14801-970 Araraquara, SP, Brazil \\ b University of São Paulo - USP, Department of Electrical and Computer Engineering, 13566-590 São Carlos, SP, Brazil \\ c CNR-Istituto di Nanotecnologia (NANOTEC) - PLasMI Lab, Via Amendola 122/D, 70126 Bari, Italy \\ ${ }^{d}$ Federal University of São Paulo - UNIFESP, Exact and Earth Sciences Department, 09913-030 Diadema, SP, Brazil
}

\section{A R T I C L E I N F O}

\section{Article history:}

Received 27 December 2017

Received in revised form 5 March 2018

Accepted 5 March 2018

Available online 7 March 2018

\section{Keywords:}

Spark discharge

LIBS

Fertilizer analysis

Phosphorus determination

\begin{abstract}
A B S T R A C T
A spark discharge system was coupled to laser-induced breakdown spectroscopy (SD-LIBS) to improve sensitivity in the determination of P in fertilizers. A LIBS system consisting of a Q-switched Nd:YAG laser operating at $1064 \mathrm{~nm}$ and emitting pulses at $50 \mathrm{~mJ}$ with a fluence of $70 \mathrm{~J} \mathrm{~cm}^{-2}$ at the focal point was used. Results of preliminary experiments suggested that the most appropriate wavelength to measure $P$ was the $P(I)$ line at $214.9 \mathrm{~nm}$, which did not show interferences by Fe, $\mathrm{Cu}$ and $\mathrm{Zn}$. The electrical discharge was provided by a homemade highvoltage electronic circuit consisting of two cylindrical tungsten electrodes at the optimized output voltage of $4.5 \mathrm{kV}$, with tips arranged at the optimal distances of $4 \mathrm{~mm}$ between them and $2 \mathrm{~mm}$ above the sample surface. To minimize the expected matrix effects calibration standards of $\mathrm{P}_{2} \mathrm{O}_{5}$ in the range of 4.8 and $33.3 \%$ were prepared by mixing various amounts of a phosphate rock reference material (SRM-120c) with a mixture of $\mathrm{CaCO}_{3}$, $\mathrm{CaSO}_{4},\left(\mathrm{NH}_{2}\right)_{2} \mathrm{CO}$ and $\mathrm{KCl}$ at a 1:1:1:1 mass ratio. The calibration curves obtained at a $4.5 \mathrm{kV}$ SD-LIBS output voltage showed correlation coefficients $\geq 0.993, \mathrm{RSD} \leq 8 \%$ and $\mathrm{LOQ} 5.3 \%(\mathrm{~m} / \mathrm{m}) \mathrm{P}_{2} \mathrm{O}_{5}$. Data obtained by analyzing commercial samples by the proposed system were in good agreement, at $95 \%$ confidence level, with those obtained by using high-resolution continuum-source flame atomic absorption spectroscopy.
\end{abstract}

(c) 2018 Elsevier B.V. All rights reserved.

\section{Introduction}

Phosphorus (P) plays an important role in several plant processes such as energy generation, photosynthesis, several enzymatic and redox reactions, carbohydrate metabolism and nitrogen fixation [1,2]. Being native soil P supplies generally poor [3], fertilizers derived from phosphate rocks are largely used in agriculture. About 40 billion tons of phosphate fertilizers were consumed in the world in the year 2000, and about 60 billion tons are expected to be used in 2040 [1,2,4]. Thus, the development of simple, modern, rapid and rugged methods for P determination in fertilizers is relevant in the context of the increasing food demand by a growing global population and decreasing non-renewable resources of phosphate rocks.

In the last years, laser-induced breakdown spectroscopy (LIBS) has been employed for P determination in phosphate ores [5,6] mineral fertilizers $[7,8]$ and organic mineral fertilizers $[9,10]$. Although the low resolution of simple LIBS systems has limited initially their applications,

\footnotetext{
* Corresponding author.

E-mail address: anchieta@iq.unesp.br (J.A.G. Neto).
}

various methods have been suggested to laser-induced breakdown spectroscopy signal enhancement [11] such as dual-pulse LIBS $[9,12,13]$, multi-pulse LIBS [14,15], spatial [16] or magnetic [17] confinement, spark discharge [18-31]. A detailed discussion on fundamentals on each enhancement method described above can be found in the review of Y. Li et al. [11]. Among other strategies to enhance sensitivity are the use of CCD detector and high fluence [8], intensified CCD detector [5,6], resonance LIBS [29], laser-induced fluorescence LIBS [32]. However, most of these approaches require additional laser devices and/or optical arrangements that often increase the system complexity and costs.

Regarding SD, it is a simple and low-cost method to overcome the low energies of lasers by reheating the plasma so increasing emission intensities [11]. In the last decade many efforts have been conducted to increase the performances of spark-assisted LIBS analysis. These include the development of fast discharge circuits [25,29], optimization of the temperature and electronic density of reheated plasma [30] and of the voltage and capacitance of the spark discharge [27], and use of electric pulses for molecular and isotopic analysis [31]. In particular, spark-assisted LIBS has been used for analysis of soil [18,19,23,25-27,30], silicon [24,29], Al [22,31], Cu [22], gypsum [21], 
metallurgical slag [21], lime, rock, $\mathrm{CaF}_{2}, \mathrm{CaO}$ [31]. But the determination of $\mathrm{P}$ in fertilizers is scarce in the current literature.

Thus, the aim of this work was to test the best performance conditions of a homemade spark discharge system to be associated to a low energy LIBS system for the determination of $\mathrm{P}$ in commercial fertilizers.

\section{Materials and methods}

\subsection{Instrumentation}

A LIBS system consisting of a Q-switched Nd:YAG laser (Quantel, Big Sky Ultra 50, Bozeman, USA) emitting at $1064 \mathrm{~nm}$ and operating at a maximum power energy of $50 \mathrm{~mJ}$ with a pulse duration of $20 \mathrm{~ns}$, a laser spot diameter of about $300 \mu \mathrm{m}$ and an ablation laser fluence of $\sim 70 \mathrm{~J} \mathrm{~cm}^{-2}$ was used in this work. The plasma emission was collected at an angle of $45^{\circ}$ with respect to the laser beam by using an optical fiber bundle, and then addressed to four spectrometers Ocean Optics (HR2000+, Dunedin, USA) featuring an optical resolution of $0.1 \mathrm{~nm}$ (full width at half maximum) and a spectral range from 200 to $630 \mathrm{~nm}$. The integration time and the Q-Switched delay used for spectra acquisition were $1 \mathrm{~ms}$ and $1 \mu$ s, respectively.

Individual samples were placed in an automatic sample holder able to move in the ' $x$-y' directions under the control of the analyst. The sampling chamber included an inner video camera able to monitor the entire process. Experiments were performed in air and ambient pressure. Thirty spectra were acquired at different positions of each calibration standard and sample. After spectral baseline correction performed by a Whittaker filter on MATLAB $®$ (R2010a) and PLS Toolbox, a software Microsoft Excel ${ }^{\circledR}$ (2016) was used for data (peak intensities) treatment.

The spark discharge was obtained using two cylindrical pure tungsten electrodes $(100 \times 2.6 \mathrm{~mm})$ with tips arranged at a distance of $4 \mathrm{~mm}$ between them and $2 \mathrm{~mm}$ above the sample surface (Fig. 1). The laser beam was focused on the sample surface in the middle of the two electrode tips. The homemade high-voltage electronic circuit consisted of simple components, i.e. a primary circuit of the transformer, a secondary circuit and a voltage divider. The first circuit consisted of an on/off switch, a signal lamp indicator, overcurrent protection and a potentiometer allowing the operator to adjust the desired voltage output from the source to the transformer. The voltage ratio of the transformer was approximately $1: 1.35$, which increased the $60 \mathrm{~Hz}$ and $127 \mathrm{~V}$ of the source to a maximum of approximately $171 \mathrm{~V}$. The sinusoidal output voltage of the transformer was then multiplied and rectified by a Walton-Cockroft circuit consisting of twenty-eight $1 \mu \mathrm{F} / 250 \mathrm{~V}$

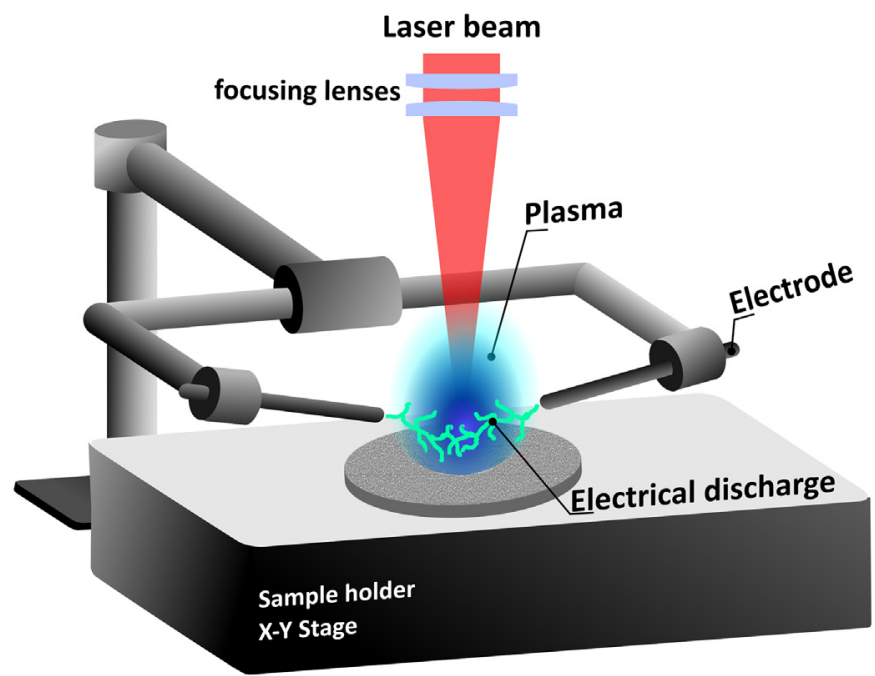

Fig. 1. Scheme of the experimental set up of the SD-LIBS system employed for $P$ determination. capacitors and 1N4007 diodes, which converted the $171 \mathrm{~V} / 60 \mathrm{~Hz}$ sinusoidal signal to a DC signal of $4500 \mathrm{~V}$. As the high currents due to the high voltage might interfere in the circuit, a $1 \mathrm{Ohm}$-resistance was connected to the high voltage terminal, which was, in turn, connected to a resistive voltage divider that allowed to lower the DC voltage signal by a factor of 100 to yield a value that could be measured safely and easily by a multimeter.

As the comparative technique, an Analytik Jena ContrAA 300 highresolution continuum source flame atomic absorption spectrometer (Jena, Germany) equipped with a xenon short-arc lamp XBO 301 (GLE, Berlin, Germany) operating at the nominal power of $300 \mathrm{~W}$ and in the hot-spot mode as the continuum radiation source was used. The instrument was equipped with a compact, high-resolution doubleEchelle grating monochromator featuring a spectral band width $<2 \mathrm{pm}$ per pixel in the far ultraviolet range, and a charge-coupled device (CCD) array detector. All measurements $(\mathrm{n}=3)$ were done according to instrumental conditions published elsewhere [33].

\subsection{Reagents, samples and procedures}

High-purity water (resistivity $18.2 \Omega \mathrm{M} \cdot \mathrm{cm}$ ) obtained from a Millipore Rios $5 \circledR$ reverse osmosis and a Millipore Milli- $Q^{\text {TM }}$ Academic $®$ deionizer system (Bedford, USA) and Merck Suprapur ${ }^{\circledR}$ nitric and hydrochloric acids (Darmstadt, Germany) were used to prepare all solutions.

Matrix-matched solid standards containing 4.8, 11.9, 22.9, 27.8 and $33.34 \%(\mathrm{~m} / \mathrm{m}) \mathrm{P}_{2} \mathrm{O}_{5}$ were prepared by mixing proper amounts of the SRM-120c phosphate rock reference material (National Institute of Standard and Technology, Gaithersburg, USA) and a combined reagent containing $\left(\mathrm{NH}_{2}\right)_{2} \mathrm{CO}$ (Merck), $\mathrm{CaCO}_{3}$ (Synth, Diadema, Brazil), $\mathrm{CaSO}_{4}$ (Merck, Darmstadt, Germany) and $\mathrm{KCl}$ (Mallinckrodt, Xalostoc, Mexico) (mass ratio of $1: 1: 1: 1$ ). The combined reagent was employed as the blank.

Six phosphate fertilizer samples containing phosphorus in the $6-17 \%(\mathrm{~m} / \mathrm{m}) \mathrm{P}_{2} \mathrm{O}_{5}$ range (values declared on the label of samples, Table 1) were acquired from local market in Araraquara-SP, Brazil. Samples, solid standards and blank were ground manually in an agate mortar, homogenized and dried at $105{ }^{\circ} \mathrm{C}$ (up to a constant weight). A portion of about $300 \mathrm{mg}$ was compacted using the Solab SL - 10/15 hydraulic press (Piracicaba, Brazil) by applying a pressure of $7.5 \mathrm{t} \mathrm{cm}^{-2}$ for $3 \mathrm{~min}$ to obtain disc-shaped pellets of approximately $13-\mathrm{mm}$ diameter for further analysis.

For comparison results, all samples were also analyzed $(\mathrm{n}=3)$ by HR-CS FAAS. Samples were pretreated by using a microwave-assisted acid-digestion system. Masses of $200 \mathrm{mg}$ of samples and the SRM120c were accurately weighted and transferred to microwave flasks followed by $2.25 \mathrm{~mL}$ of $65 \%(\mathrm{~m} / \mathrm{m}) \mathrm{HNO}_{3}, 0.75 \mathrm{~mL}$ of $32 \%(\mathrm{~m} / \mathrm{m}) \mathrm{HCl}$, $1 \mathrm{~mL}$ of $30 \%(\mathrm{v} / \mathrm{v}) \mathrm{H}_{2} \mathrm{O}_{2}$ (Merck, Darmstadt, Germany) and $2 \mathrm{~mL}$ of deionized water. The closed-vessels were inserted into the cavity of the Multiwave Anton Paar microwave oven (Graz, Austria) and the heating program was run: $15 \mathrm{~min}$ from 0 at $1000 \mathrm{~W}$ and $10 \mathrm{~min}$ at $1000 \mathrm{~W}$

Table 1

Results (mean $\pm \mathrm{SD})(\mathrm{n}=3$ ) for P determination in the SRM standard and fertilizer samples $\left(\% \mathrm{~m} / \mathrm{m} \mathrm{P}_{2} \mathrm{O}_{5}\right)$ by SD-LIBS and HR-CS-FAAS.

\begin{tabular}{|c|c|c|c|c|c|c|}
\hline \multirow{2}{*}{$\begin{array}{l}\text { Sample } \\
04-14-08^{c}\end{array}$} & \multicolumn{3}{|c|}{ ED-LIBS $^{a}$} & \multicolumn{3}{|c|}{ HR-CS FAAS $^{\mathrm{b}}$} \\
\hline & 15.0 & \pm & 1.0 & 14.6 & \pm & 0.4 \\
\hline $09-06-09^{c}$ & 6.8 & \pm & 0.6 & 6.5 & \pm & 0.2 \\
\hline $08-12-10^{c}$ & 10.8 & \pm & 0.3 & 11.1 & \pm & 0.4 \\
\hline $08-09-09^{c}$ & 9.7 & \pm & 0.6 & 9.2 & \pm & 0.1 \\
\hline $00-17-00^{c}$ & 16.4 & \pm & 0.7 & 17.2 & \pm & 0.4 \\
\hline $00-17-00^{c}$ & 16.6 & \pm & 0.3 & 17.3 & \pm & 0.5 \\
\hline SRM- $120 c^{d}$ & 33.0 & \pm & 2.0 & 33.6 & \pm & 0.4 \\
\hline \multicolumn{7}{|c|}{$\begin{array}{ll}\text { a } & \text { LOQ }=5.3 \% \\
\text { b } & \text { LOQ }=4.1 \% \\
c & \text { N. K\% }\end{array}$} \\
\hline
\end{tabular}


followed by $20 \mathrm{~min}$ of cooling. After digesting and cooling, the resulting digests were transferred to polypropylene flasks, and the volume was made up to $30 \mathrm{~mL}$ with deionized water.

All solutions were stored in high-density polypropylene bottles (Nalgene, Rochester, NY, USA). Plastic bottles and glassware materials were cleaned by soaking in $10 \%(\mathrm{v} / \mathrm{v}) \mathrm{HNO}_{3}$ at least $24 \mathrm{~h}$, and then rinsed abundantly in deionized water before use.

\section{Results and discussion}

The first experiments aimed to identify the most appropriate LIBS emission line of $\mathrm{P}$ to be used for analysis. The SD-LIBS spectra measured at a $4.5 \mathrm{kV}$ output on the sample 04-14-08 showed a number of $\mathrm{P}$ (I) lines at 213.6, 214.9 and 215.4, 253.4 and $253.6 \mathrm{~nm}$, which were much more intense than those of the corresponding LIBS spectra (an example of sample spectra is shown in Fig. 2). Further, some emission lines of Fe (II) at 253.4, 253.9 and $254.2 \mathrm{~nm}$ [34] were present due to Fe naturally present in the samples. To confirm the presence of Fe lines, the SD-LIBS spectrum of the San Joaquin Soil Standard SRM 2709a (certified values: $3.36 \pm 0.07 \% \mathrm{Fe} ; 0.0688 \% \mathrm{P}$ ) was measured and compared to that of sample 04-14-08 (Fig. 3). Intense Fe emissions at 253.4, 253.7, 253.9 and $254.2 \mathrm{~nm}$ were present in the standard, whereas no Fe lines were observed in the $213-216 \mathrm{~nm}$ interval (Fig. 3). These results suggested that P (I) lines at 253.4 and $253.6 \mathrm{~nm}$ should be avoided for P determination in fertilizers when using a $0.1 \mathrm{~nm}$ resolution spectrometer.

Further, the concentrations of Fe previously determined by HR-CS FAAS in all samples examined fell in the range from 0.14 to $1.08 \%(\mathrm{~m} /$ $\mathrm{m}$ ), i.e. they were about 2-3 times lower than that in the SRM 2709a standard. This result confirmed that a negligible Fe interference would be expected on $\mathrm{P}$ (I) lines located in the $213-216 \mathrm{~nm}$ spectral range. However, the $\mathrm{P}(\mathrm{I})$ line at $213.6 \mathrm{~nm}$ might overlap with the $\mathrm{Cu}(\mathrm{I})$ line at $213.5 \mathrm{~nm}$ and $\mathrm{Zn}$ (I) line at $213.9 \mathrm{~nm}$. As $\mathrm{Cu}$ and $\mathrm{Zn}$ might be present at low concentration in some phosphate fertilizer formulations [35], the $\mathrm{P}$ (I) line at $213.6 \mathrm{~nm}$ might be interfered by these elements, thus this line should also be excluded from the $P$ analysis. In conclusion, although both P (I) lines at $215.4 \mathrm{~nm}$ and $214.9 \mathrm{~nm}$ presented suitable selectivity, the latter was chosen for further experiments due to its higher (about 3.4) relative sensitivity.

The possible effect of the components of the tungsten electrodes used to obtain the spark was evaluated by comparing the SD-LIBS spectrum of sample 04-14-08 to that of the blank (Fig. 2). Apparently, the emissions from these components were close to the background, which confirmed the suitability of these electrodes to be used even at the maximum voltage applied $(4.5 \mathrm{kV})$.

The influence of SD voltage on the P (I) emission intensity at $214.9 \mathrm{~nm}$ was tested by varying it in the range $0-4.5 \mathrm{kV}$ at a fixed

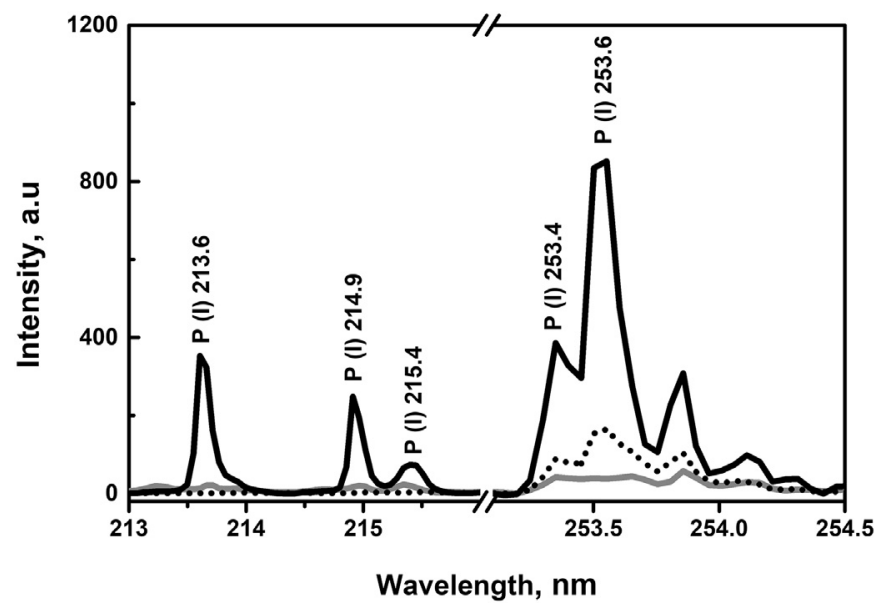

Fig. 2. LIBS (....) and SD-LIBS (-) spectra of the sample 04-14-08 and SD-LIBS (-) spectrum of the blank. SD-LIBS spectra were obtained at a $4.5 \mathrm{kV}$ output.

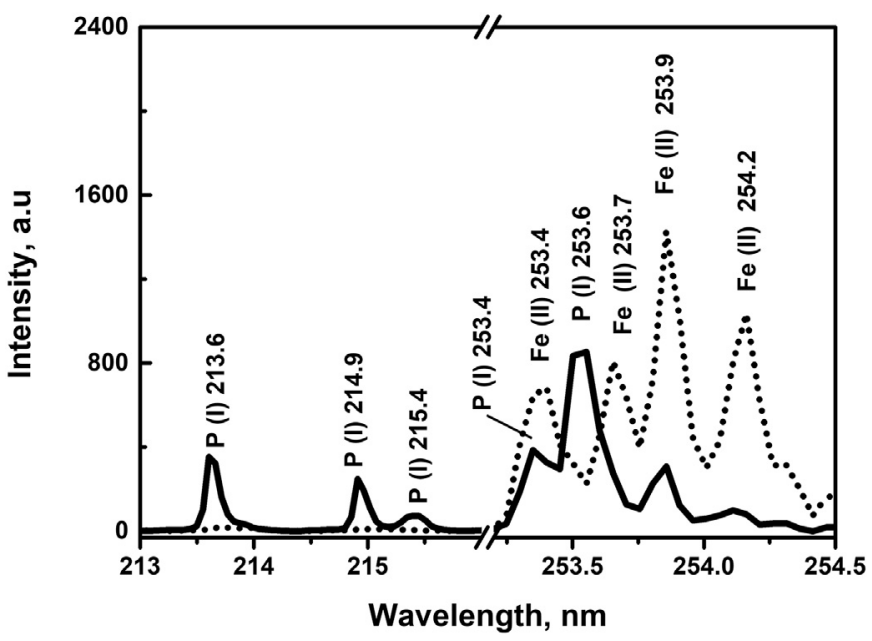

Fig. 3. SD-LIBS spectra obtained at a $4.5 \mathrm{kV}$ output of sample 04-14-08 (-) and SRM 2709a standard (

distance of $4 \mathrm{~mm}$ between electrode tips. The emission intensity increased linearly with increasing the voltage up to $4.5 \mathrm{kV}$ (Fig. 4), with a typical RSD $\leq 7 \%$, which could be ascribed to the increase of plasma temperature caused by the reheating electric pulse $[11,23,27,29]$. Thus, the voltage of $4.5 \mathrm{kV}$ was chosen to analyze the samples.

The effect of the distance between electrode tips on the performance of the SD circuit was evaluated by measuring the intensity of the $\mathrm{P}$ (I) line at $214.9 \mathrm{~nm}$ at distances of 2, 3, 4, 5 and $6 \mathrm{~mm}$ (Fig. 5). The emission intensity appeared to increase up to a tip distance of $4 \mathrm{~mm}$ and then decrease. At the shortest distances, the ionic species produced in the plasma favored the flowing of a fast current through the circuit, which caused the electrical arc produced to enter only partially into the bulk plasma, so that the plasma reheating contribution was low. At distances greater than $4 \mathrm{~mm}$ more collisions were favored in the electron path between electrodes, which reduced the energy of electrons so that they were less efficient in exciting/ionizing the species present in the plasma. These findings were in agreement with the Paschen law, which states that the breakdown voltage at a certain distance between electrode tips is a function of the product of the gas pressure and the distance [36]. Thus, all further experiments were performed at a distance of $4 \mathrm{~mm}$ between electrode tips.

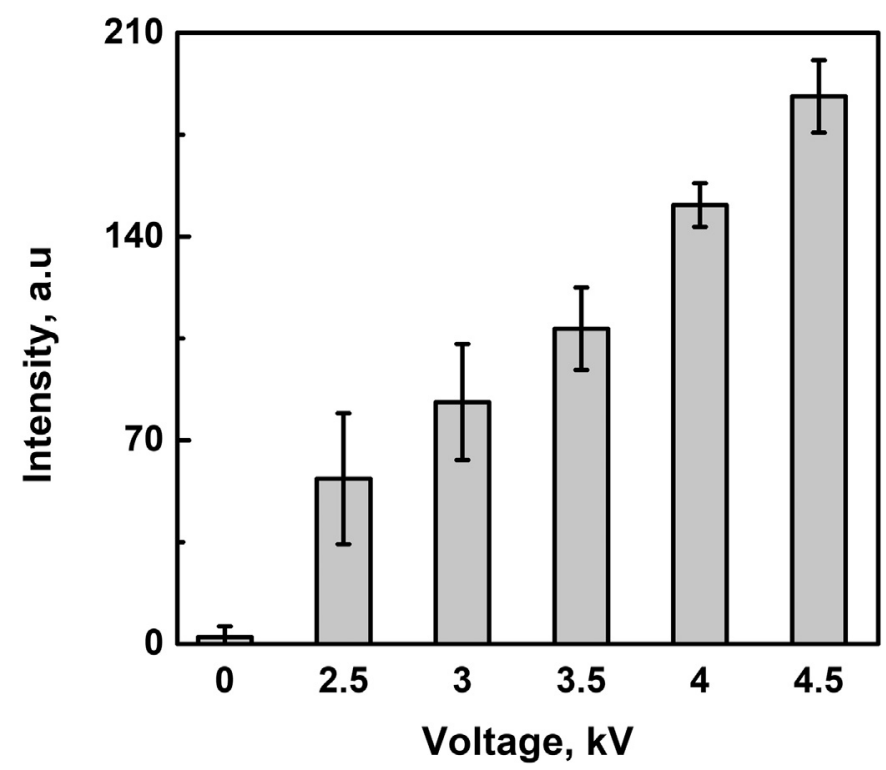

Fig. 4. Emission intensity of $\mathrm{P}(\mathrm{I})$ at line $214.9 \mathrm{~nm}$ as a function of output applied voltage 


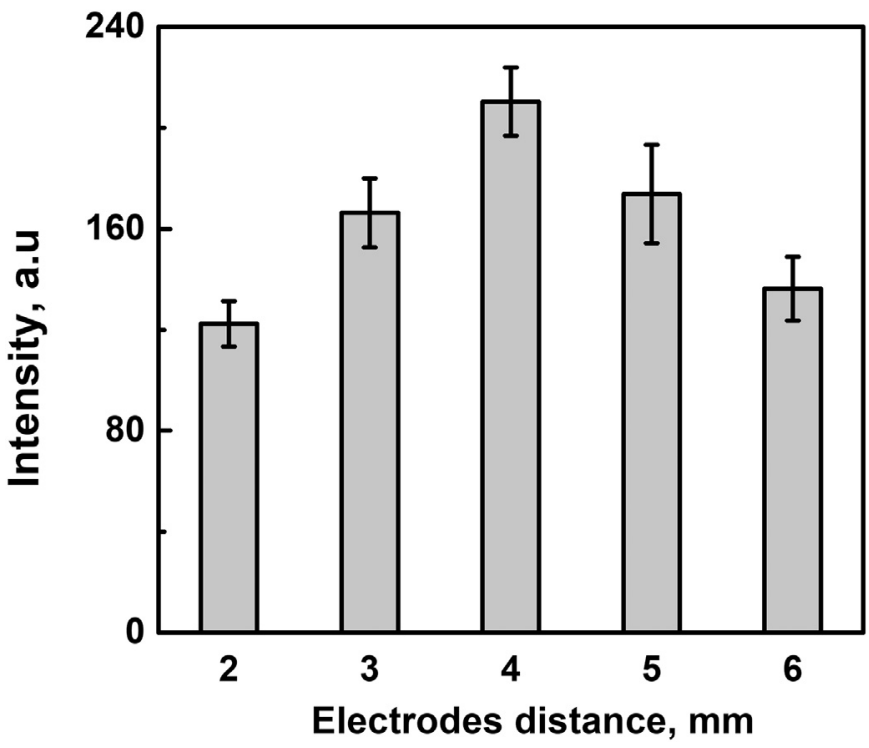

Fig. 5. Emission intensity of the $\mathrm{P}(\mathrm{I})$ line at $214.9 \mathrm{~nm}$ as a function of the electrode tips distance at a $4.5 \mathrm{kV}$ output.

After the optimization of SD operational parameters, calibration curves were firstly constructed by using $\mathrm{NaHPO}_{4}$ as solid standard. Although this standard provided satisfactory linear correlation coefficients, the accuracy was poor, i.e. the P content measured by SD-LIBS in some samples was almost twice than that measured by HR-CSFAAS, probably because the matrix-effects influenced markedly the interaction laser-samples and processes of plasma formation [5,37]. Thus, considering that most commercial phosphate fertilizers contain phosphate rocks, $\mathrm{NaHPO}_{4}$ was replaced by the phosphate rock standard SRM-120c to yield solid standards containing the same percentage of $\mathrm{P}_{2} \mathrm{O}_{5}$ as indicated above in Section 2.2.

The calibration curves constructed using the SAM-120c standard featured correlation coefficients higher than 0.993 between the $\mathrm{P}$ (I) emission at $214.9 \mathrm{~nm}$ and the $\mathrm{P}_{2} \mathrm{O}_{5}$ concentration in \% $(\mathrm{m} / \mathrm{m})$ measured by HR-CS-FAAS. The RSD values were $\leq 8 \%$ and the limit of quantification (LOQ) calculated by the $10 \sigma /$ Slope, where $\sigma$ is the standard deviation of 10 measurements of the blank, was $5.3 \%(\mathrm{~m} / \mathrm{m}) \mathrm{P}_{2} \mathrm{O}_{5}$, which is equivalent to $2.3 \%(\mathrm{~m} / \mathrm{m})$ P. Although the LOQ value obtained by SD-LIBS can be considered fully acceptable for P measurement in commercial fertilizers [6,35], that LOQ can be improved by using the procedure of summation of spectra to increase sensibility. In particular, by employing ten cumulative spectra on each site of the sample surface, the $\mathrm{LOQ}$ was reduced to $3 \%(\mathrm{~m} / \mathrm{m}) \mathrm{P}_{2} \mathrm{O}_{5}$. In particular, due to limitations of the low energy LIBS system employed in this work, the data processing associated to the spectral sum was performed using an external software (Microsoft office excel 2016). However, this procedure was not required to analyze the samples used in this work.

The level contents of $P$ in fertilizer samples analyzed by the proposed SD-LIBS method were in agreement at 95\% confidence with those obtained by HR-CS FAAS comparative technique (Table 1). For further accuracy evaluation, the phosphate rock standard SRM-120c was also analyzed by SD-LIBS, and the P concentration measured was in agreement (at 95\% confidence) with the certified value of the SRM (Table 1).

\section{Conclusions}

In conclusion, the SD-assisted LIBS system showed to be an efficient tool to increase the sensitivity of P measurement in phosphate fertilizers. The coupling SD-LIBS did not require any synchronization tool between the laser pulse and the electrical discharge, i.e. the latter was the result of charged conducting species in the laser-induced plasma. Further, the SD circuit represents a simple, easily operated and low-cost acquisition and maintenance system that can be coupled to any LIBS apparatus showing promising for further applications to other analytes and matrices.

\section{Acknowledgments}

The authors are grateful to the Fundação de Amparo à Pesquisa do Estado de São Paulo (FAPESP) (Grant\# 2004/14212-0) for supporting financially this work, and to the Conselho Nacional de Desenvolvimento Científico e Tecnológico (Grant\# 2015/24757-9) (CNPq) for fellowship granted to A.L.V. (Grant\# 141977/2016-7) and research ship granted to J.A.G.N. (Grant\# 303255/2013-7) and D.S.J. (Grant\# 311085/20148). The authors are also grateful to R.A.C.A. of the Department of Electrical and Computer Engineering of the Engineering School of São Carlos USP, for providing the materials and instruments used for the design of the high voltage circuit.

\section{References}

[1] S. Suh, S. Yee, Phosphorus use-efficiency of agriculture and food system in the US, Chemosphere 84 (2011) 806-813.

[2] C.P. Vance, Symbiotic nitrogen fixation and phosphorus acquisition. Plant nutrition in a world of declining renewable resources, Plant Physiol. 127 (2001) 390-397.

[3] K. Ashley, D. Cordell, D. Mavinic, A brief history of phosphorus: from the philosopher's stone to nutrient recovery and reuse, Chemosphere 84 (2011) 737-746.

[4] C.P. Vance, C. Uhde-Stone, D.L. Allan, Phosphorus acquisition and use: critical adaptations by plants for securing a nonrenewable resource, New Phytol. 157 (2003) 423-447.

[5] S. Rosenwasser, G. Asimellis, B. Bromley, R. Hazlett, J. Martin, T. Pearce, A. Zigler, Development of a method for automated quantitative analysis of ores using LIBS, Spectrochim. Acta B 56 (2001) 707-714.

[6] G. Asimellis, A. Giannoudakos, M. Kompitsas, Phosphate ore beneficiation via determination of phosphorus-to-silica ratios by Laser Induced Breakdown Spectroscopy, Spectrochim. Acta B 61 (2006) 1253-1259.

[7] S. Yao, J. Lu, J. Li, K. Chen, J. Li, M. Dong, Multi-elemental analysis of fertilizer using laser-induced breakdown spectroscopy coupled with partial least squares regression, J. Anal. At. Spectrom. 25 (2010) 1733-1738.

[8] B.S. Marangoni, K.S.G. Silva, G. Nicolodelli, G.S. Senesi, J.S. Cabral, P.R. Villas-Boas, C.S. Silva, P.C. Teixeira, A.R.A. Nogueira, V.M. Benites, D.M.B.P. Milori, Phosphorus quantification in fertilizers using laser induced breakdown spectroscopy (LIBS): a methodology of analysis to correct physical matrix effects, Anal. Methods 8 (2015) 78-82.

[9] G. Nicolodelli, G.S. Senesi, I.L. de Oliveira Perazzoli, B.S. Marangoni, V. De Melo Benites, D.M.B.P. Milori, Double pulse laser induced breakdown spectroscopy: A potential tool for the analysis of contaminants and macro/micronutrients in organic mineral fertilizers, Sci. Total Environ. 565 (2016) 1116-1123.

[10] G.S. Senesi, R.A. Romano, B.S. Marangoni, G. Nicolodelli, P.R. Villas-Boas, V.M. Benites, D.M.B.P. Milori, Laser-induced breakdown spectroscopy associated with multivariate analysis applied to discriminate fertilizers of different nature, J. Appl. Spectrosc. 84 (2017) 923-928.

[11] Y. Li, D. Tian, Y. Ding, G. Yang, K. Liu, C. Wang, X. Han, A review of laser-induced breakdown spectroscopy signal enhancement, Appl. Spectrosc. Rev. 53 (2018) 1-35.

[12] K.H. Kurniawan, M.O. Tija, K. Kagawa, Review of laser-induced plasma, its mechanism, and application to quantitative analysis of hydrogen and deuterium, Appl. Spectrosc. Rev. 49 (2014) 323-434.

[13] G. Nicolodelli, G.S. Senesi, A.C. Ranulfi, B.S. Marangoni, A. Watanabe, V.M. Benites, P.P.A. de Oliveira, P. Villas-Boas, D.M.B.P. Milori, Double-pulse laser induced breakdown spectroscopy in orthogonal beam geometry to enhance line emission intensity from agricultural samples, Microchem. J. 133 (2017) 272-278.

[14] N. Jedlinszki, G. Galbács, An evaluation of the analytical performance of collinear multi-pulse laser induced breakdown spectroscopy, Microchem. J. 97 (2011) 255-263.

[15] A. De Giacomo, M. Dell'Aglio, D. Bruno, R. Gaudiuso, O. De Pascale, Experimental and theoretical comparison of single-pulse and double-pulse laser induced breakdown spectroscopy on metallic samples, Spectrochim. Acta B 63 (2008) 805-816.

[16] A.M. Popov, F. Colao, R. Fantoni, Enhancement of LIBS signal by spatially confining the laser-induced plasma, J. Anal. At. Spectrom. 24 (2009) 602-604.

[17] L. Cheng, G. Xun, L. Qi, C. Song, Spectral enhancement of laser-induced breakdown spectroscopy in external magnetic field, Plasma Sci. Technol. 17 (2015) 919-922.

[18] K.X. Li, W.D. Zhou, Q.M. Shen, Z.J. Ren, B.J. Peng, Laser ablation assisted spark induced breakdown spectroscopy on soil samples, J. Anal. At. Spectrom. 25 (2010) 1475-1481.

[19] M.V. Belkov, V.S. Burakov, A. De Giacomo, V.V. Kiris, S.N. Raikov, N.V. Tarasenko, Comparison of two laser-induced breakdown spectroscopy techniques for total carbon measurement in soils, Spectrochim. Acta B 64 (2009) 899-904.

[20] H. Sobral, A. Robledo-Martinez, Signal enhancement in laser-induced breakdown spectroscopy using fast square-pulse discharges, Spectrochim. Acta B 124 (2016) 67-73.

[21] S. Eschlböck-Fuchs, P.J. Kolmhofer, M.A. Bodea, J.G. Hechenberger, N. Huber, R. Rössler, J.D. Pedarnig, Boosting persistence time of laser-induced plasma by electric arc discharge for optical emission spectroscopy, Spectrochim. Acta B 109 (2015) 31-38. 
[22] O.A. Nassef, H.E. Elsayed-Ali, Spark discharge assisted laser induced breakdown spectroscopy, Spectrochim. Acta B 60 (2005) 1564-1572.

[23] L.I. Kexue, W. Zhou, Q. Shen, J. Shao, H. Qian, Signal enhancement of lead and arsenic in soil using laser ablation combined with fast electric discharge, Spectrochim. Acta B 65 (2010) 420-424.

[24] W. Zhou, K. Li, Q. Shen, Q. Chen, J. Long, Optical emission enhancement using laser ablation combined with fast pulse discharge, Opt. Express 18 (2010) 2573-2578.

[25] W. Zhou, K. Li, X. Li, H. Oian, J. Shao, X. Fang, P. Xie, W. Liu, Development of a nanosecond discharge-enhanced laser plasma spectroscopy, Opt. Lett. 36 (2011) 2961-2963.

[26] X. Li, W. Zhou, K. Li, H. Qian, Z. Ren, Laser ablation fast pulse discharge plasma spectroscopy analysis of Pb, Mg and Sn in soil, Opt. Commun. 285 (2011) 54-58.

[27] W. Zhou, K. Li, H. Qian, Z. Ren, Y. Yu, Effect of voltage and capacitance in nanosecond pulse discharge enhanced laser-induced breakdown spectroscopy, Appl. Opt. 51 (2012) 42-48.

[28] D. Wu, L. Sun, P. Liu, R. Hai, H. Ding, Enhancement of laser-induced breakdown spectroscopic signals in a liquid jet with glow discharge, Appl. Spectrosc. 72 (2018) 225-233.

[29] W. Zhou, X. Su, H. Qian, K. Li, X. Li, Y. Yu, Z. Ren, Discharge character and optical emission in a laser ablation nanosecond discharge enhanced silicon plasma, J. Anal. At. Spectrom. 28 (2013) 702-710
[30] X.F. Li, W.D. Zhou, Z.F. Cui, Temperature and electron density of soil plasma generated by LA-FPDPS, Front. Phys. 7 (2012) 721-727.

[31] A.A. Bol'shakov, X. Mao, R.E. Russo, Spectral emission enhancement by an electric pulse for LIBS and LAMIS, J. Anal. At. Spectrom. 32 (2017) 657-670.

[32] S. Laville, C. Goueguel, H. Loudyi, F. Vidal, M. Chaker, M. Sabsabi, Laser-induced fluorescence detection of lead atoms in a laser-induced plasma: an experimental analytical optimization study, Spectrochim. Acta B 64 (2009) 347-353.

[33] M.A. Bechlin, F.M. Fortunato, R.M. Da Silva, E.C. Ferreira, J.A. Gomes Neto, A simple and fast method for assessment of the nitrogen-phosphorus-potassium rating of fertilizers using high-resolution continuum source atomic and molecular absorption spectrometry, Spectrochim. Acta B 101 (2014) 240-244.

[34] [26] NIST Atomic Spectra Database, https://physics.nist.gov/PhysRefData/ASD/lines_ form.html (accessed 2 March 2018).

[35] P.E. Trani, A.L. Trani, Fertilizantes: cálculo de fórmulas comerciais, Instituto Agronômico, Campinas, 2011 (29 pp., Série Tecnologia APTA. Boletim Técnico IAC, 208).

[36] A.P. Henry, College Physics, New York, 13th ed., 1947.

[37] D.A. Cremers, L.J. Radziemski, Handbook of Laser-Induced Breakdown Spectroscopy, 2nd ed. John Wiley \& Sons, Tucson, 2013. 